\title{
Overexpression of UHRF1 promotes silencing of tumor suppressor genes and predicts outcome in hepatoblastoma
}

\author{
Alexander Beck ${ }^{1 \dagger}$, Franziska Trippel $^{1 \dagger}$, Alexandra Wagner $^{1}$, Saskia Joppien ${ }^{1}$, Max Felle $^{2}$, Christian Vokuhl ${ }^{3}$, \\ Thomas Schwarzmayr ${ }^{4,5}$, Tim M. Strom $^{4,5}$, Dietrich von Schweinitz ${ }^{1}$, Gernot Längst ${ }^{2}$ and Roland Kappler ${ }^{1^{*}}$ (D)
}

\begin{abstract}
Background: Hepatoblastoma $(\mathrm{HB})$ is the most common liver tumor of childhood and occurs predominantly within the first 3 years of life. In accordance to its early manifestation, HB has been described to display an extremely low mutation rate. As substitute, epigenetic modifiers seem to play an exceptional role in its tumorigenesis, which holds promise to develop targeted therapies and establish biomarkers for patient risk stratification.

Results: We examined the role of a newly described protein complex consisting of three epigenetic regulators, namely E3 ubiquitin-like containing PHD and RING finger domain 1 (UHRF1), ubiquitin-specific-processing protease 7 (USP7), and DNA methyltransferase 1 (DNMT1), in HB. We found the complex to be located on the promoter regions of the pivotal HB-associated tumor suppressor genes (TSGS) HHIP, IGFBP3, and SFRP1 in HB cells, thereby leading to strong repression through DNA methylation and histone modifications. Consequently, knockdown of UHRF1 led to DNA demethylation and loss of the repressive H3K9me2 histone mark at the TSG loci with their subsequent transcriptional reactivation. The observed growth impairment of HB cells upon UHRF1 knockdown could be attributed to reduced expression of genes involved in cell cycle progression, negative regulation of cell death, LIN28B signaling, and the adverse 16-gene signature, as revealed by global RNA sequencing. Clinically, overexpression of UHRF1 in primary tumor tissues was significantly associated with poor survival and the prognostic high-risk 16-gene signature.
\end{abstract}

Conclusion: These findings suggest that UHRF1 is critical for aberrant TSG silencing and sustained growth signaling in $\mathrm{HB}$ and that UHRFI overexpression levels might serve as a prognostic biomarker and potential molecular target for HB patients.

Keywords: UHRF1, Hepatoblastoma, DNMT1, USP7, Histone methylation, DNA methylation, Epigenetic silencing, Tumor suppressor genes, Biomarker

\section{Background}

Hepatoblastoma $(\mathrm{HB})$ is the most common liver tumor of childhood, with the majority of cases occurring in children under the age of 3 years [1]. Over the last four decades, advances in treatment protocols comprising chemotherapy and surgery drastically improved outcome of $\mathrm{HB}$ patients from a $30 \%$ to a roughly $80 \%$ overall

\footnotetext{
* Correspondence: roland.kappler@med.uni-muenchen.de

${ }^{\dagger}$ Equal contributors

${ }^{1}$ Department of Pediatric Surgery, Dr. von Hauner Children's Hospital, Ludwig-Maximilians-University Munich, Lindwurmstr. 2a, 80337 Munich, Germany

Full list of author information is available at the end of the article
}

survival rate [2]. However, there is still a relevant subgroup of high-risk patients presenting with distant metastasis, vascular invasion, advanced tumor stages, or unfavorable histology, whose outcome remains poor [3, 4]. In recent years, molecular markers have been shown to improve $\mathrm{HB}$ risk stratification and the effort to find robust markers that can predict outcome in $\mathrm{HB}$ patients continues $[5,6]$.

Molecularly, the development of this aggressive embryonal tumor remains largely unknown. Exome sequencing of primary $\mathrm{HB}$ revealed a surprisingly low mutation rate of only 2.9 mutations per tumor on average, and 
there is evidence that epigenetic dysregulation plays a key role in HB development and progression [7]. Aberrant epigenetic silencing of tumor suppressor genes (TSGs) and overexpression of epigenetic regulators of gene silencing such as histone deacetylases (HDACs) appear to be important drivers of this disease [8]. In particular, the silencing of the TSGs hedgehog-interacting protein $(H H I P)$, insulin-like growth factor-binding protein 3 (IGFBP3), and secreted frizzled-related protein 1 (SFRP1) have been shown to deregulate pivotal pathways of embryonic development, thus promoting HB pathogenesis $[9,10]$. While there is evidence that DNA methylation and repressive histone modifications are responsible for aberrant silencing of those genes, the molecular mechanisms through which those epigenetic changes are conveyed and maintained remain largely elusive.

We recently discovered a protein complex that is involved in aberrant DNA methylation and repression of TSGs in a human colon adenocarcinoma cell line [11]. The complex comprises three subunits, namely DNA methyltransferase 1 (DNMT1), ubiquitin-specificprocessing protease 7 (USP7), and E3 ubiquitin-like containing PHD and RING finger domain 1 (UHRF1) (Fig. 1a). Those three proteins are capable of forming a trimeric repression complex through their specific binding domains and carry out distinct functions within the complex. DNMT1 effectively methylates DNA on CpG islands and maintains DNA methylation patterns throughout the cell cycle [12, 13]. USP7 has been shown to enhance DNMT1 function and to stabilize UHRF1 levels by preventing its degradation [11]. While UHRF1 has no chromatin or DNA methylation activities itself, it functions as a crucial guide for DNMT1 and USP7 by binding to both via its SET- and RING-associated (SRA) domain. Through this domain, UHRF1 is also capable of recognizing hemi-methylated DNA, thereby recruiting the complex to hemi-methylated $\mathrm{CpGs}$ and maintaining DNA methylation throughout the genome [14].

Furthermore, UHRF1 is involved in histone modification, as it recognizes the repressive histone $\mathrm{H} 3$ lysine 9 methylation mark (H3K9me2) via its tandem tudor domain (TTD), thus facilitating the maintenance of this mark and the formation of heterochromatin $[15,16]$. UHRF1 is also able to form a complex with HDAC1, another facilitator of histone-driven gene repression [17]. Because of this dynamic crosstalk between histone and DNA modifications, UHRF1 occupies a unique role when it comes to long-term silencing of genes [18].

Overexpression and dysregulation of UHRF1 are common features in a variety of human malignancies, and UHRF1-mediated silencing of TSGs appears to drive tumorigenesis in a disease-specific context [19]. In fact, UHRF1 is so widely overexpressed in solid tumors that

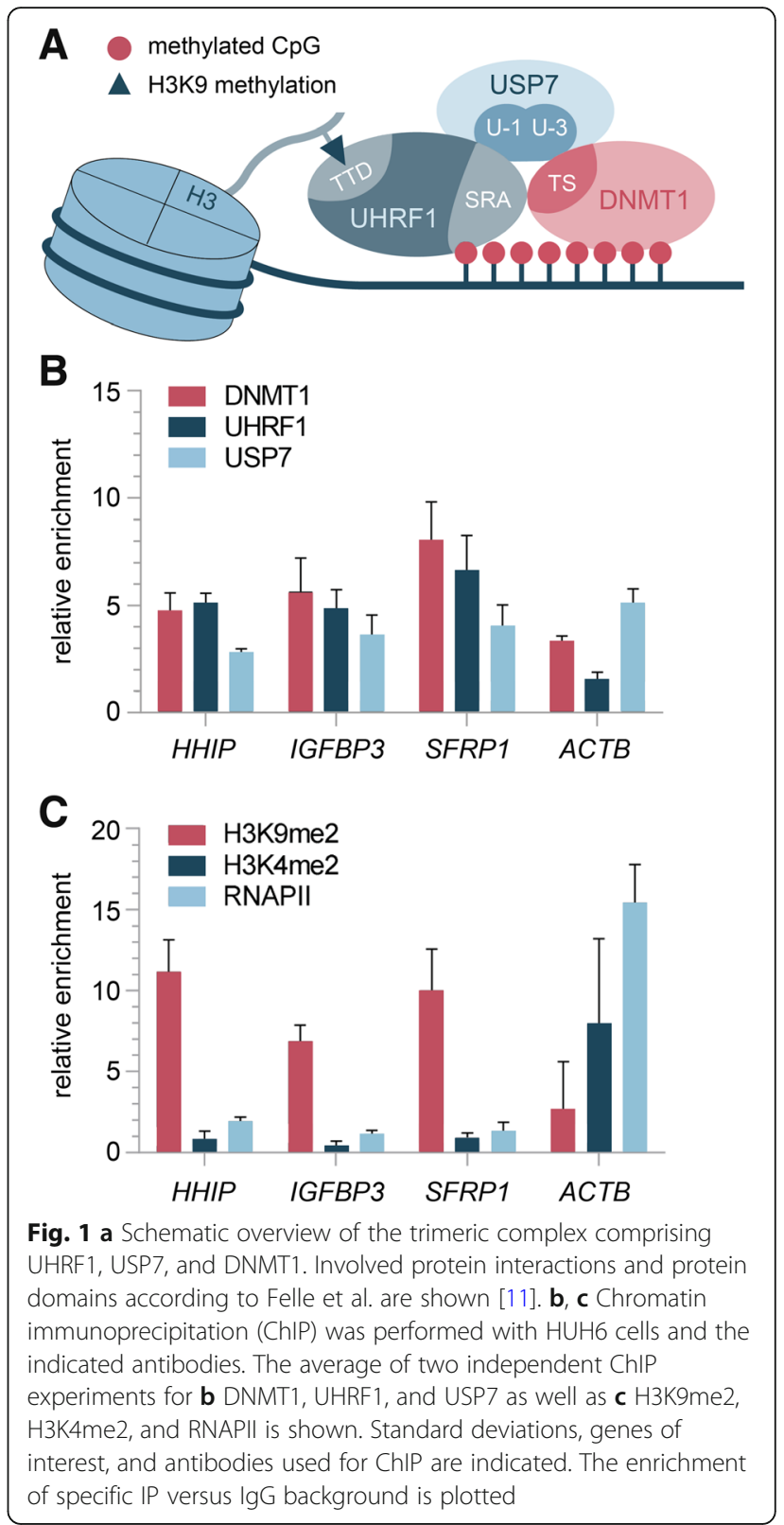

it has been suggested as a universal biomarker for cancer [20]. However, the challenge lies in deciphering the disease-specific context in which UHRF1 conveys its oncogenic properties.

Here, we show the trimeric UHRF1 complex to be located on the promoter regions of HHIP, IGFBP3, and SFRP1 in HB cells, thereby leading to strong repression of those pivotal HB-associated TSGs through DNA methylation and histone modifications. Knockdown of UHRF1 results in demethylation of promoter regions, loss of the repressive H3K9me2 mark, and subsequent re-expression of the abovementioned TSGs in HB cells. Furthermore, we show that UHRF1 knockdown facilitates proliferation impairment and global transcriptional 
changes in HB cells. Finally, we reveal overexpression of UHRF1 in primary HB to be associated with poor patient outcome and established risk stratification criteria. These findings suggest that UHRF1 is critical for aberrant TSG silencing and sustained growth signaling in $\mathrm{HB}$ and that UHRF1 expression levels might serve as a prognostic biomarker for $\mathrm{HB}$ patients.

\section{Methods}

\section{Patients}

A total of 40 liver tumor specimens were obtained from pediatric patients undergoing surgical resection in our department. Matching normal liver was available from seven patients. Written informed consent was obtained from each patient, and the study protocol was approved by the Committee of Ethics of the Ludwig Maximilian University of Munich.

\section{Cell culture and transfection}

The human hepatoblastoma cell lines HUH6 (Japanese Collection of Research Bioresources, JCRB, Osaka, Japan), HepT1 (provided by Dr. T. Pietsch), and HepG2 (ATCC, Manassas, USA) were cultured under standard conditions in RPMI 1640 growth media (Invitrogen, Carlsbad, CA, USA), supplemented with $10 \%$ fetal calf serum, $100 \mathrm{U} / \mathrm{mL}$ penicillin, and $100 \mu \mathrm{g} / \mathrm{mL}$ streptomycin at $37{ }^{\circ} \mathrm{C}$ in a $5 \% \mathrm{CO}_{2}$ incubator. For knockdown experiments, $2 \times 10^{6}$ cells were electroporated for $10 \mathrm{~ms}$ at $350 \mathrm{~V}$ with either siRNA targeting UHRF1 (siRNA \#s26553, Applied Biosystems, Darmstadt, Germany) or appropriate negative control siRNA (siGENOMEN non targeting siRNA \#1, Thermo Scientific, Karlsruhe, Germany) in a final concentration of 80 pmol.

\section{RNA isolation, reverse transcription, and qRT-PCR}

Total RNA was isolated $48 \mathrm{~h}$ after transfection using TriReagent (Sigma-Aldrich, Steinheim, Germany) according to the manufacturer's instructions. Two micrograms of total RNA was reverse transcribed into cDNA using random hexamer primer (Roche Diagnostics, Penzberg, Germany) and SuperScriptII Reverse Transcriptase (Invitrogen, Karlsruhe, Germany). PCR amplifications on the Master cycler ep gradient (Eppendorf, Hamburg, Germany) using iTaq-SYBR Green-Supermix (Bio-Rad, Hercules, CA) were performed in doublets as described previously [21]. The following primer pairs $\left(5^{\prime}->3^{\prime}\right.$ orientation) were used: UHRF1, CTCCACGTCCAGGCCG, TGGAGTTCATCTGGACCACG; HHIP, TGTACATCATTCTTGGTGATGGG, AGCCGTAGCA CTGAGCCTGT; IGFBP3, GTCCAAGCGGGAGACAGAATAT, CCTGGGACTCAGCACATTGA; SFRP1, CA TGACGCCGCCCAAT, GATGGCCTCAGATTTCAAC TCG; and TBP, GCCCGAAACGCCGAATAT, CCGTG GTTCGTGGCTCTCT. Data were normalized to the expression level of the housekeeping gene TATA-Boxbinding protein (TBP). For calculation of the relative mRNA expression level, the $\Delta \Delta C T$ method was used and expressed as fold change relative to the corresponding control sample [22].

\section{Western blot analysis}

Forty-eight hours after transfection, cells were harvested with ice-cold lysis buffer $(0.2 \mathrm{M} \mathrm{KCl}, 0.03 \mathrm{M}$ Tris, $\mathrm{pH}$ 7.25) containing proteinase inhibitor cocktail (Roche Diagnostics). Lysates were boiled in equal volumes of loading buffer (125 mM Tris- $\mathrm{HCl}, \mathrm{pH} 6.8,4 \%$ SDS, 20\% glycerol, and $10 \%$ ß-mercaptoethanol), and $20 \mu \mathrm{g}$ of protein was separated in a $8-12 \%$ gradient Tris-glycine gradient gel (Novex, San Diego, CA, USA) under reducing conditions and transferred to nitrocellulose membranes (GE Healthcare, Piscataway, USA). Afterwards, membranes were blocked at room temperature with PBS containing $0.1 \%$ Tween and $5 \%$ non-fat dry milk to block non-specific binding for $2 \mathrm{~h}$. Membranes were incubated with primary antibodies (rabbit anti-human $B$-actin, Cell Signaling Technology, Danvers, USA; mouse anti-human UHRF1, generously provided by Dr. C. Bronner, Institute of Genetics and Molecular and Cellular Biology, Strasbourg, France) at $4{ }^{\circ} \mathrm{C}$ overnight, followed by a 1-h incubation with the corresponding secondary antibodies (goat anti-rabbit-HRP and goat anti-mouse-HRP, both Dako Cytomation, Hamburg, Germany) at room temperature. For chemiluminescent detection, the ECL Plus Western detection kit (GE Healthcare) was used. Protein bands were detected by autoradiography using the high-performance autoradiography Hyperfilm ${ }^{\text {Tm }}$ MP (GE Healthcare).

\section{Methylation-specific polymerase chain reaction (MSP)}

Genomic DNA was extracted by phenol/chloroform after proteinase $\mathrm{K}$ treatment. Two micrograms of purified genomic DNA was used for bisulfite-mediated conversion of unmethylated cytosine using the Epitec Bisulfite Kit (Qiagen, Hilden, Germany). For MSP, bisulfite-treated DNA was amplified with primers specific for the methylated $(\mathrm{M})$ and unmethylated $(\mathrm{U})$ promoter region of either the HHIP (from -230 to $-87 \mathrm{bp}$ upstream of the transcriptional start site), the IGFBP3 (from -180 to $-13 \mathrm{bp}$ upstream of the transcriptional start site), or the SFRP1 (from + 36 to +175 bp downstream of the transcriptional start site) gene. As a control for MSP, we used in vitro methylated genomic DNA that has been treated for $4 \mathrm{~h}$ with $40 \mathrm{U}$ CpG methyltransferase (M. SssI), NEBuffer2 (10×), and SAM (1:20) at $37{ }^{\circ} \mathrm{C}$, followed by heat inactivation for $20 \mathrm{~min}$ at $65{ }^{\circ} \mathrm{C}$. For MSP, we used $1 \mathrm{U}$ hot start Taq polymerase (Thermo Scientific, Schwerte, Germany), $1 \times$ hot start Taq buffer, $2 \mathrm{mM}$ dNTPs, $1.5 \mathrm{mM} \mathrm{MgCl}_{2}, 100 \mathrm{ng}$ 
bisulfite-treated DNA, and $500 \mathrm{nM}$ of the following forward and reverse primers $\left(5^{\prime}->3^{\prime}\right.$ orientation) and annealing temperatures: HHIP-M-F, AGTAGTCGGGTATGTTCG GAATTTTC and HHIP-M-R, GAACCTTCGAAACCAAC CTCG at $53{ }^{\circ} \mathrm{C}$; IGFBP3-M-F, GCGAGTTTCGAGTTG TACGTTTTC and IGFBP3-M-R, GCCGACCGCTATATA AAAACCG at $61{ }^{\circ} \mathrm{C}$; SFRP1-M-F, TTTGTAGTTTTCGGA GTTAGTGTCGC and SFRP1-M-R, CGACCCTCGACC TACGATCG at $58{ }^{\circ} \mathrm{C}$; HHIP-U-F, TTGTAGTAGTTGGG TAGTTTTGGAATTTTT and HHIP-U-R, AAACCTTAA AACCAACCTCAAAA at $53{ }^{\circ} \mathrm{C}$; IGFBP3-U-F, TTGGG TGAGTTTTGAGTTGTATGTTTTT and IGFBP3-U-R, AAACACACCAACCACTATATAAAAACCAAA at $61{ }^{\circ} \mathrm{C}$; and SFRP1-U-F, TTTTGTAGTTTTTGGAGTTAGTGTT GTGTG and SFRP1-U-R, CAATAACAACCCTCAACCTA CAATCAA at $58{ }^{\circ} \mathrm{C}$. MSP primer design was accomplished using Methyl Primer Express (Applied Biosystems) using the following criteria: CpG percentage $>55 \%$, observed/expected CpG $>65 \%$, and CpG length $>300$ bp. MSP reactions were carried out at the following conditions: hot start at $94{ }^{\circ} \mathrm{C}$ for $4 \mathrm{~min}$, followed by 38 cycles of $94{ }^{\circ} \mathrm{C}$ for $30 \mathrm{~s}$, gene-specific annealing temperature (see above) for $30 \mathrm{~s}, 72{ }^{\circ} \mathrm{C}$ for $45 \mathrm{~s}$, and final extension at $72{ }^{\circ} \mathrm{C}$ for $10 \mathrm{~min}$. One percent agarose gel electrophoresis was performed to visualize DNA amplicons.

\section{Pyrosequencing}

For pyrosequencing, $100 \mathrm{ng}$ bisulfite-treated DNA was first amplified in a PCR reaction, using $1 \mathrm{U}$ hot start Taq polymerase (Thermo Scientific), $1 \times$ hot start Taq buffer, $2 \mathrm{mM}$ dNTPs, $1.5 \mathrm{mM} \mathrm{MgCl}_{2}, 100 \mathrm{ng}$ bisulfite-treated DNA, and $500 \mathrm{nM}$ of the following forward and reverse primers $\left(5^{\prime}->3^{\prime}\right.$ orientation) and annealing temperatures: HHIP-F, GGGAGGAGAGAGGAGTTT and HHIP-R, AACCAACCTCCAAAATACTAAACC at $55^{\circ} \mathrm{C}$; IGFBP3F, TGGTTTTTTGAGATTTAAATGTAAGTTAGA and IGFBP3-R, ATCACCCCAATCACTCCTA at $57{ }^{\circ} \mathrm{C}$; SFRP1-F, GGAGTTAGAGATTAGTTTGGTTAATATGG and SFRP1-R, AAAAACCTAAATCATACTTACAACC at 54 ${ }^{\circ} \mathrm{C}$; and LINE1-F and LINE1-R (assay X58075, Qiagen) at $55{ }^{\circ} \mathrm{C}$. PCR reactions were run at $95{ }^{\circ} \mathrm{C}$ for $4 \mathrm{~min}$ and 45 cycles of $95{ }^{\circ} \mathrm{C}$ for $20 \mathrm{~s}$, gene-specific annealing temperature (see above) for $20 \mathrm{~s}$, and $72{ }^{\circ} \mathrm{C}$ for $30 \mathrm{~s}$. PCR products were sequenced with the corresponding sequencing primers HHIP-Seq, TTTAGGATTGAGTTTTTGTT TTAAG; IGFBP3-Seq, TTGGGTTATTTAGGTTTTATAT AG; and SFRP1-Seq, GGTAAGAGGTTGTAATTTTAG TTAT using PyroMark Gold Q24 reagents (Qiagen).

\section{Chromatin immunoprecipitation (ChIP)}

$8.0 \times 10^{6}$ HUH6 cells were transfected with siRNA against UHRF1 or corresponding non-target siRNA and subsequently seeded in $15-\mathrm{cm}$ plates. After $48 \mathrm{~h}$, the protein-DNA complexes were cross-linked with $1 \%$ formaldehyde for $10 \mathrm{~min}$. The cross-linked reaction was quenched with Glycine Stop-Fix Solution for $5 \mathrm{~min}$. Cell lysis, enzymatic digest (5 min), chromatin immunoprecipitation with $2 \mu \mathrm{g}$ of antibody against di-methylated H3K4, di-methylated H3K9, RNA-Pol2 (all from Active Motif, La Hulpe, Belgium), and mouse IgG (Santa Cruz, Heidelberg, Germany), respectively, as well as final elution, cross-link reversal, and proteinase $\mathrm{K}$ digestion, were performed according to the manufacturer's protocol (catalog no. 53009, Active Motif). Chromatin samples were subjected to a DNA clean-up step using the QIAquick PCR Purification kit (QIAGEN). Real-time PCR was performed on purified DNA from each of the ChIP reactions using primer pairs for loci of the promoter region of the HHIP gene, the IGFBP3 gene, the SFRP1 gene, and the $A C T B$ gene. Primer pairs used in this study were the following: HHIP_ChIP_F, TTCCCACCTCCT ACGGCC and HHIP_ChIP_R, TCCTCTCTCCTCCCC GCTT; IGFBP3_ChIP_F, GCTCCCTGAGACCCAAA TGTAA and IGFBP3_ChIP_R, GCTCGGCATTCGTG TGTACC; SFRP1_ChIP_F, ACGCCGTGATCCATTC CC and SFRP1_ChIP_R, CGGCTCAACACCCCTTAA AAA; and ACTB_ChIP_F, GCCAACGCCAAAACT CTCC and ACTB_ChIP_R, CAGTGCAGCATTTTT TTACCCC.

\section{Cell viability assay}

To assess cell proliferation, a 3-(4,5-dimethyl-2-thiazolyl)-2,5-diphenyl-2 $H$-tetrazolium bromide (MTT)-based protocol was used. Directly after electroporation, cells were seeded at a density of 2500 (HUH6 and HepT1) or 5000 (HepG2) cells per well in a 96-well format (Nunc, Wiesbaden, Germany) in $100 \mu \mathrm{L}$ RPMI medium. Ten microliters of MTT labeling agent ( $5 \mathrm{mg} / \mathrm{mL}$, Sigma) was added to each well and incubated at $37^{\circ} \mathrm{C}$ for $4 \mathrm{~h}$. One hundred microliters of a SDS- $\mathrm{HCl}$ solution was added to each well and mixed thoroughly. Microplate was incubated overnight at $37^{\circ} \mathrm{C}$ in a humidified chamber. The absorbance of this colorimetric reaction was quantified on the GENios reader (Tecan, Männedorf, Switzerland) by measuring at a wavelength of $595 \mathrm{~nm}$.

\section{Cell migration assay}

Transfected HB cells were seeded into 12-well plates and grown to complete confluency. A wound of approximately $1 \mathrm{~mm}$ was inflicted to cell monolayers with a pipette tip. The wells were washed with PBS to remove detached cells and incubated at $37{ }^{\circ} \mathrm{C}$ for a maximum time of $120 \mathrm{~h}$. Images were taken every $24 \mathrm{~h}$ after scratching, and the wound widths were measured with ImageJ (Rasband, W.S., ImageJ, US National Institutes of Health, Bethesda, MD, USA). 


\section{RNA sequencing}

Total RNA of siUHRF1 and control transfected HUH6 cells was quality checked and quantitatively measured using the RNA 6000 nanokit on a 2100 Bioanalyzer (Agilent Technologies, Santa Clara, CA, USA). Coding transcriptomes were enriched from $1 \mu \mathrm{g}$ total RNA in solution with TruSeq non-stranded RNA v2 kits (Illumina, San Diego, CA, USA) and sequenced as $100 \mathrm{bp}$ paired-end runs on a HiSeq2500 system (Illumina) generating 35-79 million mapped reads. The STAR aligner $\mathrm{v}$ 2.4.2a [23] with modified parameter settings (-twopassMode $=$ Basic) is used for split-read alignment against the human genome assembly hg19 (GRCh37) and UCSC knownGene annotation. To quantify the number of reads mapping to annotated genes, we use HTseq-count v0.6.0 [24]. The R Bioconductor package DESeq2 [25] with modified standard settings (no fold-change shrinkage) is used to investigate differences in gene expression.

\section{Gene set enrichment analysis}

Enrichment analysis of gene ontology and hallmark gene sets was performed by using the top 1000 differentially expressed genes of the RNA sequencing results as input for the latest version of the Molecular Signatures Database (MSigDB) developed by the Broad Institute [26]. Gene sets were selected by statistical significance $(p<$ $0.05)$ and the calculated representation factor. The representation factor is the number of overlapping genes divided by the expected number of overlapping genes drawn from two independent groups. A representation factor $>1$ indicates more overlap than expected of two independent groups, and a representation factor $<1$ indicates less overlap than expected.

\section{Statistical analysis}

Data were expressed as mean \pm standard deviation or \pm standard error of the mean and statistically subjected to Student's unpaired $t$ test. Kaplan-Meier estimates of specific survival time in the various groups were compared using the log-rank Mantel-Cox test. Gene expression correlation was performed with GraphPad Prism version 6.0 using Spearman's rank correlation. A level of $p<0.05$ was considered to be significant.

\section{Results}

UHRF1 repression complex binds to HB-specific TSG promoter regions

We used chromatin immunoprecipitations (ChIP) to identify enrichment of the UHRF1 repression complex (Fig. 1a) on chromatin of HB cells. We found all three complex partners, namely UHRF1, DNMT1, and USP7, to be enriched on the promoter regions of the HBspecific TSGs HHIP, IGFBP3, and SFRP1 (Fig. 1b). Furthermore, ChIP analysis of the repressive H3K9me2 and activating H3K4me2 histone marks showed enrichment of H3K9me2, while H3K4me2 was not detected (Fig. 1c). The enrichment for RNA polymerase II (RNAPII) was negligible, indicating very low or no active transcription of those genes. Subsequent methylation-specific polymerase chain reaction (MSP) revealed hypermethylated promoter regions of those three genes (Fig. 2a). Quantitative mRNA analysis of HHIP, IGFBP3, and SFRP1 confirmed the virtual silencing of gene expression (Fig. 2b). In sharp contrast, the housekeeping gene $A C T B$ did not show any enrichment for UHRF1 and the repressive H3K9me2 mark, but high levels of H3K4me2 and RNAPII, which was accompanied by an unmethylated promoter and a high transcriptional activity. These data suggest that the trimeric UHRF1 repression complex might play a crucial role in deep silencing of the TSGs HHIP, IGFBP3, and SFRP1 in HB via combined epigenetic mechanisms, comprising repressive histone modification and DNA methylation.

UHRF1 knockdown leads to re-expression of TSGs through epigenetic modifications

To evaluate the effect of UHRF1 depletion in HB cells, we performed knockdown experiments, which resulted in a strong reduction of UHRF1 both on the mRNA and the protein level (Fig. 3a, b). Depletion of UHRF1 led to a significant re-expression of HHIP and IGFBP3 (Fig. 3c). While the re-expression of SFRP1 was not significant,

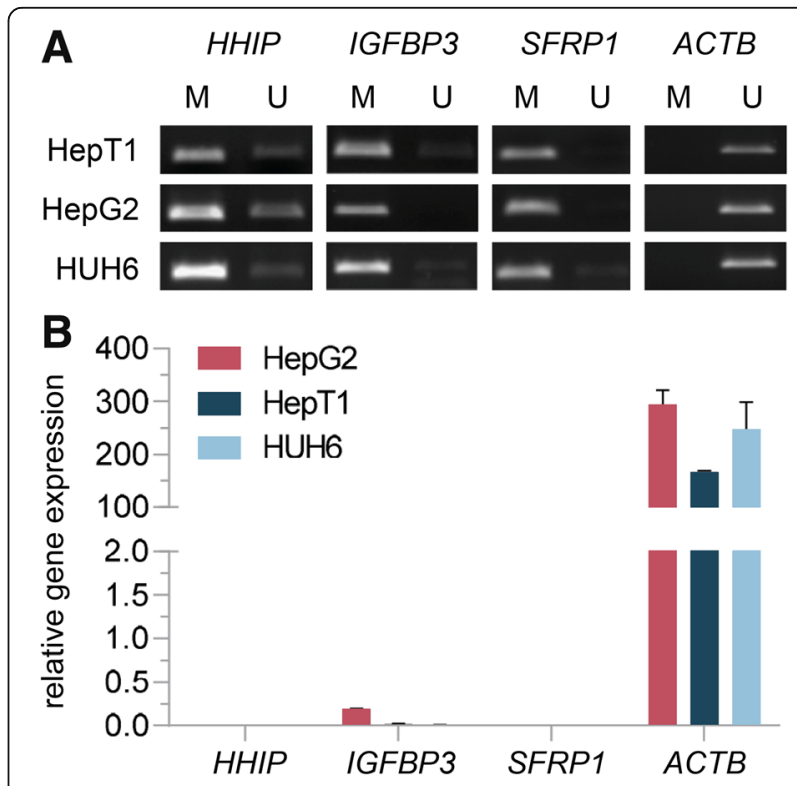

Fig. 2 a Methylation status ( $U$, unmethylated; $M$, methylated) of the HHIP, IGFBP3, SFRP1, and ACTB promoter region was determined for the indicated cell lines by MSP. Representative images of MSP experiments are shown. b Relative expression levels of indicated genes compared to the TBP housekeeping gene in three liver tumor cell lines are given. The average of three independent experiments is shown 

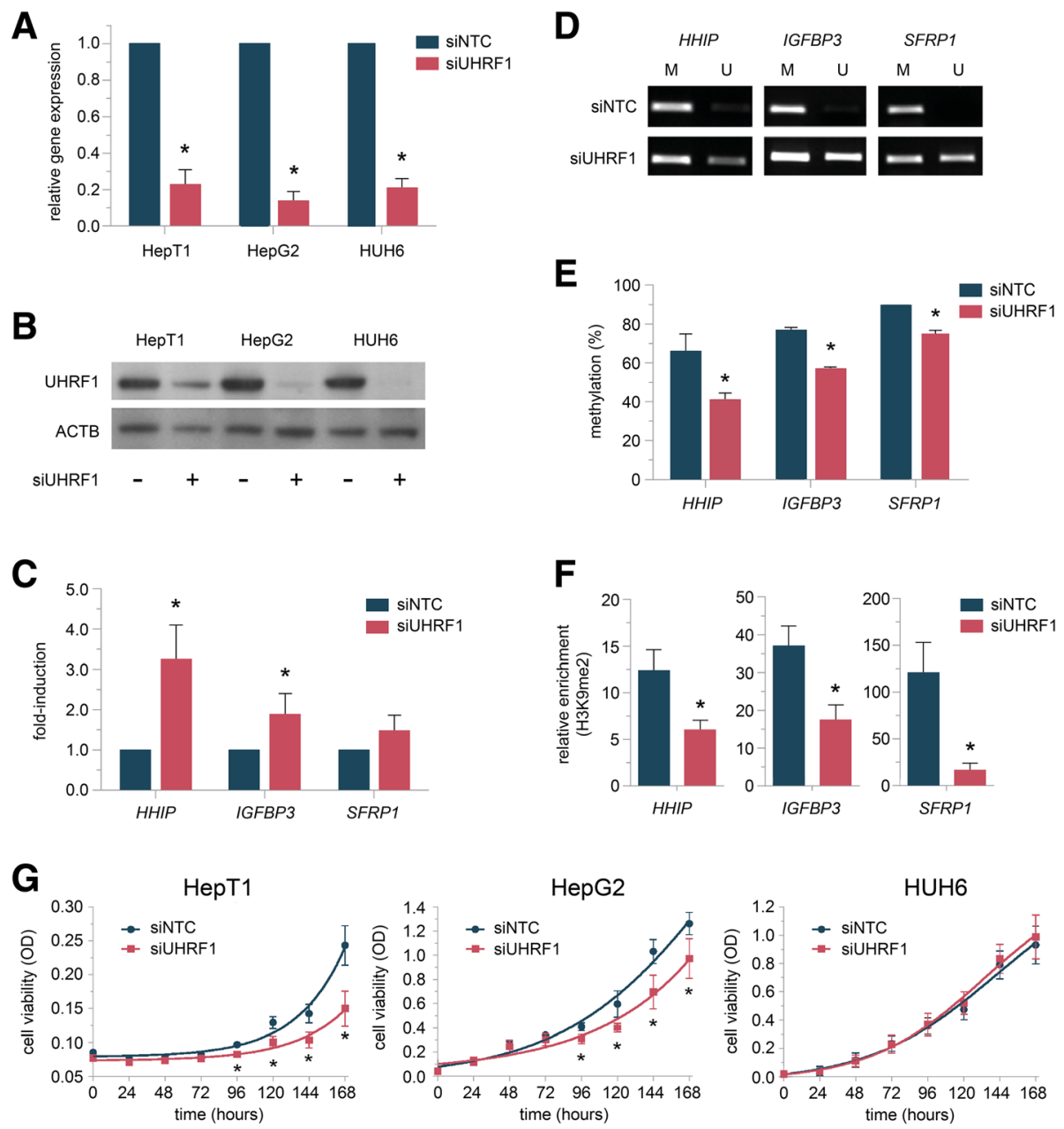

Fig. 3 a Knockdown of UHRF1 in indicated cell lines. Relative gene expression in cells transfected with siRNA against UHRF1 compared to negative control (siNTC) after $48 \mathrm{~h}$ is shown. Data represents average of three independent experiments. $\mathbf{b}$ Western blot analysis of HepT1, HepG2, and HUH6 cells $48 \mathrm{~h}$ after UHRF1 knockdown. c Relative expression levels of indicated genes in HUH6 cells $48 \mathrm{~h}$ after UHRF1 knockdown compared to negative control. The average of three independent experiments is shown. $\mathbf{d}$ Methylation status $(U$, unmethylated; $M$, methylated) of the HHIP, IGFBP3, and SFRP1 promoter region $48 \mathrm{~h}$ after UHRF1 knockdown was determined by MSP in HUH6 cells. Representative images of MSP experiments are shown. e Pyrosequencing results of promoter regions of indicated genes in HUH6 cells $48 \mathrm{~h}$ after UHRF1 knockdown of three independent experiments are shown. $\mathbf{f ~ C h I P}$ analyses in HUH6 cells $48 \mathrm{~h}$ after UHRF 1 knockdown. The relative enrichment of H3K9me2 at the indicated gene promoter regions is shown. $\mathbf{g}$ Cell viability of HepG2, HepT1, and HUH6 cells as evaluated by MTT assay at the indicated time points after UHRF1 knockdown. Values represent means \pm standard deviation of three independent experiments performed in duplicates. Statistical significance of all experiments was calculated using $t$ test $(p<0.05)$

we still observed an increase of SFRP1 expression upon UHRF1 knockdown. To further elucidate the role of the UHRF1 repression complex, we performed MSP upon knockdown of UHRF1, USP7, and DNMT1, respectively. UHRF1 knockdown led to a significant demethylation of DNA on the indicated TSG promoter regions (Fig. 3d). Quantification of those differentially methylated loci by pyrosequencing revealed a methylation decrease of 25,20 , and $15 \%$ for HHIP, IGFBP3, and SFRP1, respectively, upon UHRF1 knockdown (Fig. 3e). This decrease in methylation correlates well with the strength of TSG re-expression. In addition, UHRF1 knockdown also led to loss of the repressive H3K9me2 mark at those loci (Fig. 3f). USP7 knockdown had no significant effect on the epigenetic marks regulating those genes and also did not result in gene reactivation (data not shown). To investigate the effect of those epigenetic changes and the re-expression of TSGs on HB cell proliferation, we performed viability assays over the course of 1 week following the knockdown. UHRF1-depleted HB cells showed a significant growth reduction in two out of three cell lines over time (Fig. 3g). This data suggests a combined derepressive effect of UHRF1 knockdown on TSGs by alleviating repressive DNA and histone modifications, leading to a subsequent growth inhibition in HB cells. 
RNA sequencing reveals HB-specific transcriptional changes upon UHRF1 knockdown

In order to assess the effect of UHRF1 depletion on the transcriptome, we performed RNA sequencing of $\mathrm{HB}$ cells upon UHRF1 knockdown. As expected, we found UHRF1 to be strongly downregulated and known UHRF1-repressed target genes [27-29] to be upregulated after UHRF1 knockdown, thus emphasizing the significance of our RNA sequencing results (Fig. 4a).

To evaluate the general effect of UHRF1 depletion in $\mathrm{HB}$ cells, we then used the top 1000 differentially expressed genes as input for the analysis of gene ontology and hallmark gene sets (Fig. 4b). The analysis showed significant upregulation of genes involved in the promotion of cell differentiation and development as well as cell adhesion. Genes involved in the p53 pathway were also significantly upregulated, indicating activation of this tumor suppressive pathway upon UHRF1 knockdown. Conversely, UHRF1 depletion led to significant downregulation of genes involved in epithelial- mesenchymal transition (EMT), cell cycle progression, and negative regulation of cell death. In order to evaluate the functional effect of downregulated EMT genes of UHRF1-depleted HUH6 cells, we performed cell migration assays in the identical cell line upon UHRF1 knockdown. UHRF1-depleted cells showed a markedly slower cell migration compared to their control-transfected counterparts (Fig. 4e).

In recent years, LIN28B has been shown to play a pivotal role in $\mathrm{HB}$ initiation and progression [30]. Interestingly, we found $L I N 28 B$ and its downstream targets to be downregulated in UHRF1-depleted HB cells (Fig. 4c). In agreement with this finding, we detected upregulation of LET7 species, which are commonly suppressed by LIN28B in HB.

In $\mathrm{HB}$, a prognostic 16-gene classifier has been established that discriminates between two subclasses of tumors, the so-called $\mathrm{C} 1$ subclass that is associated with early tumor stage and favorable patient outcome and the C2 subclass that is linked to metastases, vascular
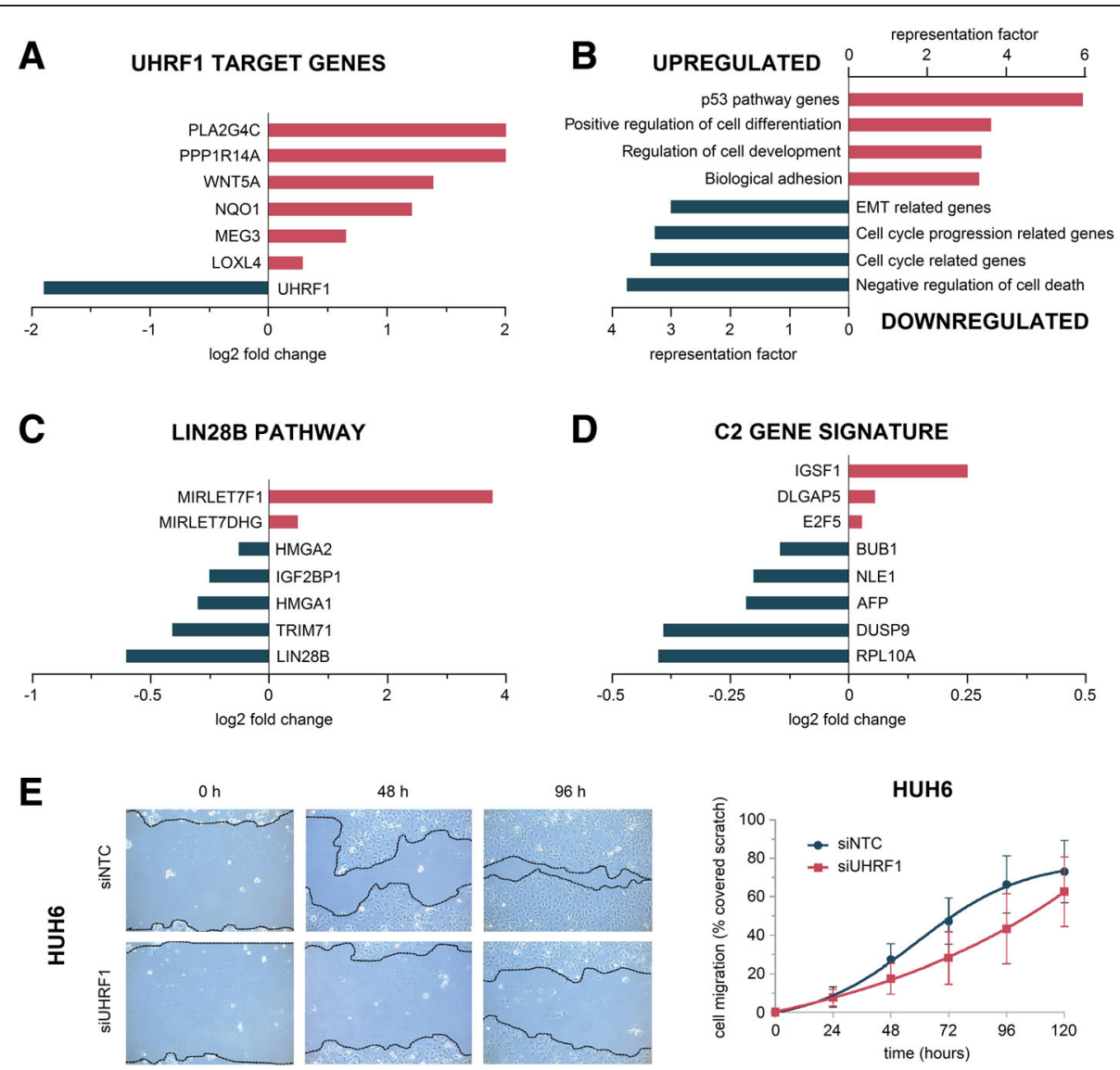

Fig. 4 RNA sequencing results upon UHRF1 knockdown in HUH6 cells. $\log _{2}$ fold change of a known UHRF1 target genes, $\mathbf{c}$ genes related to the LIN28B pathway, and $\mathbf{d}$ genes highly expressed in the adverse C2 gene signature. $\mathbf{b}$ Significant enrichment of up- or downregulated genes within the indicated gene ontology and hallmark gene sets. Enrichment is indicated by the corresponding representation factor of each gene set. e Scratch assay. Representative images of HUH6 cells transfected with siRNA against UHRF1 migrating into a cell-free space at 0, 48, and $96 \mathrm{~h}$ compared to negative control (siNTC). Quantitative evaluation of scratch closure shows the mean values of two independent experiments 
invasion, advanced tumor stage, and poor prognosis [5]. Since HB cell lines initially showed the adverse C2 expression signature, we looked for changes in the eight genes that exhibit high expression levels within the C2 signature. Notably, five of those genes were downregulated upon UHRF1 depletion (Fig. 4d), indicating a shift towards the favorable $\mathrm{C} 1$ signature, which is associated with low expression levels of those genes. Real-time qPCR analysis confirmed the downregulation of $\mathrm{C} 2$ - and LIN28B-associated genes upon UHRF1 knockdown (Additional file 1: Figure S1). These findings suggest a more global role of UHRF1 in HB that goes well beyond the activation of only a few TSGs.

\section{UHRF1 overexpressed in high-risk HB}

When we investigated the expression levels of all three subunits of the trimeric repression complex, we found only $U H R F 1$ to be overexpressed in primary tumor samples and $\mathrm{HB}$ cell lines when compared to normal liver tissue (Fig. 5a). Overexpression of UHRF1 has been reported in a variety of human malignancies and is often predictive for higher tumor stages and poor patient outcome [20]. Indeed, we found that high UHRF1 expression levels were significantly associated with poor survival in $\mathrm{HB}$ patients (Fig. 5b). Of note, when comparing UHRF1 expression levels with previously published expression levels of the tumor suppressor gene HHIP [21] in primary tumor tissues and the three cell lines, we also found a significant inverse correlation of both genes (Fig. 5c, inset).

Interestingly, we also found tumors exhibiting the high-risk $\mathrm{C} 2$ signature to be significantly associated with high expression levels of UHRF1 (Fig. 5c). Consequently, patients with vascular tumor invasion, the unfavorable embryonal histology, metastases, large PRETEXT IV, and multifocal tumors predominantly had high expression of UHRF1. These data suggest that, consistent with findings in other solid tumors, UHRF1 expression might be a prognostic marker for patients suffering from HB.

\section{Discussion}

Genetically, HB is a very simple tumor with one of the lowest mutation rates ever reported for any malignancy [31]. Characterizing HB by its genetic events has only
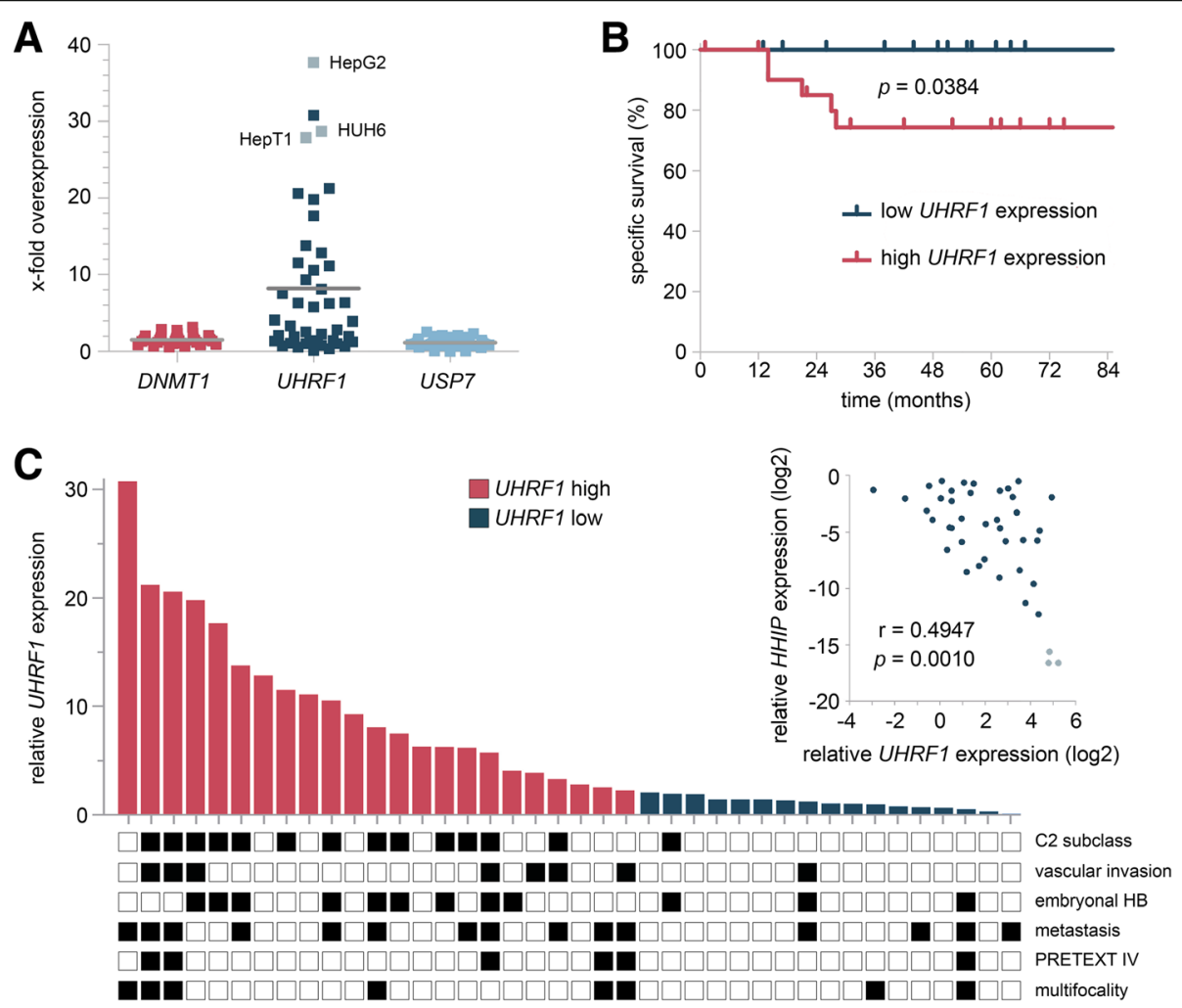

Fig. 5 a The relative expression of DNMT1, UHRFT, and USP7 in 40 primary HB and three HB cell lines (depicted in gray) compared to the mean of seven normal liver tissues are given. b Overall survival was calculated as time from diagnosis to death of the disease and is plotted for $40 \mathrm{HB}$ patients. Statistical significance was calculated using the Mantel-Cox test. c Individual UHRF1 expression levels of 40 primary HBs are shown with the occurrence of important clinicopathological characteristics depicted as black boxes below. Inset: Expression levels of UHRF1 and HHIP in primary tumor tissues (dark blue dots) and tumor cell lines (light blue dots) were plotted against each other, and Spearman's rank correlation was performed 
limited significance when it comes to risk stratification and treatment selection. In addition, genetic mutations do not explain HB tumorigenesis sufficiently and previous studies made clear that epigenetic phenomena play a key role in HB development [10, 32].

While a number of epigenetic events, such as silencing of TSGs in HB, have been described in the past, the underlying molecular mechanisms remain largely unknown. Here, we identified a trimeric repression complex that is located at HB-specific TSG sites and is capable of conveying strong transcriptional repression of those genes by combined histone and DNA modification. Knockdown of UHRF1, which appears to be the critical subunit of the trimeric complex, leads to histone and DNA demethylation, re-expression of TSGs, and growth inhibition in $\mathrm{HB}$ cells. Reduced viability upon knockdown might be a result of the downregulation of genes involved in cell cycle progression and the negative regulation of cell death. UHRF1 has been shown to be a negative regulator of the p53 tumor suppressor pathway, and growth inhibition in $\mathrm{HB}$ cells might be augmented by the activation of this pathway following UHRF1 depletion [33]. Knockdown-related upregulation of genes involved in cell differentiation and development might be a combined effect of p53 activation and the reexpression of TSGs that are capable of blocking aberrant growth signaling in HB cells. Notably, UHRF1 knockdown led to downregulation of the HB-initiating LIN28B pathway and induced a shift towards the more favorable $\mathrm{C} 1$ signature. It also led to the upregulation of genes involved in cell adhesion and downregulation of EMTrelated genes, thus promoting a more anti-metastatic phenotype. Our results strongly indicate that UHRF1 represents an interesting molecular target for novel treatment strategies in HB.

As epigenetic aberrations are potentially reversible, a number of epigenetic therapy approaches have been developed in the last few years, some of which have shown great promise in the treatment of cancer [34]. In fact, we have recently shown that HDAC inhibition constitutes a potential epigenetic treatment option for high-risk $\mathrm{HB}$ patients [8]. Targeting UHRF1 in HB holds the potential to further improve patient outcome, while reducing doses and toxicities of untargeted chemotherapeutic agents. Notably, the first in vitro studies of newly discovered UHRF1 inhibitors have shown encouraging results in several cancer cell lines [35-38].

Furthermore, UHRF1 overexpression seems to be a common feature in many malignancies and has therefore been suggested as a universal biomarker for cancer [20, 39-41]. Our systematic expression analysis of a large set of primary $\mathrm{HB}$ uncovered $U H R F 1$ overexpression to be a potential high-risk feature of $\mathrm{HB}$, as high $U H R F 1$ expression levels were significantly correlated with poor survival in $\mathrm{HB}$ patients. Moreover, the $\mathrm{C} 2$ subtype of the 16-gene signature that has been shown to predict poor prognosis in $\mathrm{HB}$ was also significantly associated with high UHRF1 expression.

\section{Conclusions}

Collectively, our findings suggest that UHRF1 is critical for aberrant epigenetic modifications and sustained growth signaling in HB. The unique capability of UHRF1 to convey repressive DNA and histone modifications highlights its potential to induce deep TSG silencing and other oncogenic events. UHRF1 therefore constitutes a promising target for novel therapeutic approaches in $\mathrm{HB}$ patients. Its overexpression in patients with high-risk features makes UHRF1 also a strong candidate for a prognostic biomarker in $\mathrm{HB}$.

\section{Additional file}

Additional file 1: Figure S1. Relative RNA expression levels of indicated genes in HUH6 cells $24 \mathrm{~h}$ after UHRF1 knockdown compared to controltransfected cells. Data were normalized to the expression level of the housekeeping gene TBP. The average of two independent knockdown experiments is shown. Statistical significance of all experiments was calculated using $t$ test $(p<0.05)$. (PNG $110 \mathrm{~kb}$ )

\section{Abbreviations}

ACTB: Beta actin; ChIP: Chromatin immunoprecipitation; CpG: Cytosinephospho-guanine; DNMT1: DNA methyltransferase 1; EMT: Epithelialmesenchymal transition; H3K4me2: Histone 3 lysine 4 di-methylation; H3K9me2: Histone 3 lysine 9 di-methylation; HB: Hepatoblastoma; HDAC: Histone deacetylase; HHIP: Hedgehog-interacting protein; IGFBP3: Insulin-like growth factor-binding protein 3; LIN28B: Lin-28 homolog B; MSP: Methylation-specific polymerase chain reaction; $\mathrm{p} 53$ : Tumor protein p53; RNAPII: RNA polymerase II; SFRP1: Secreted frizzled-related protein; SRA: SET- and RING-associated; TSG: Tumor suppressor gene; TTD: Tandem tudor domain; UHRF1: E3 ubiquitin-like containing PHD and RING finger domain 1; USP7: Ubiquitin-specific-processing protease 7

\section{Acknowledgements}

We are grateful to F. Promoli for the technical assistance, Dr. C. Bronner for generously providing the mouse anti-human UHRF1 antibody, and Dr. T. Pietsch for supplying us with the HepT1 cell line.

\section{Funding}

This work was supported by grants of the Bettina Bräu Foundation, Munich, Germany, and the Gänseblümchen-Voerde Foundation, Voerde, Germany (to RK).

\section{Availability of data and materials}

The datasets included in the present study are available from the corresponding author on a reasonable request.

\section{Authors' contributions}

$A B, F T, A W, S J$, and MF performed the experiments and analyzed data. CV and DvS provided the patient materials and clinical data and interpreted the patient data. TS and TMS set up the RNA sequencing analysis tool and generated the data. GL contributed to the design and interpretation of experiments. $A B$ and RK prepared the manuscript and figures. RK designed and supervised the study. All authors read and approved the final manuscript.

Ethics approval and consent to participate

Written informed consent was obtained from each patient, and the study protocol was approved by the Committee of Ethics of the Ludwig Maximilian University of Munich (no. 431-11). 


\section{Consent for publication}

Not applicable

\section{Competing interests}

The authors declare that they have no competing interests.

\section{Publisher's Note}

Springer Nature remains neutral with regard to jurisdictional claims in published maps and institutional affiliations.

\section{Author details}

'Department of Pediatric Surgery, Dr. von Hauner Children's Hospital, Ludwig-Maximilians-University Munich, Lindwurmstr. 2a, 80337 Munich, Germany. ${ }^{2}$ Department of Biochemistry III, University Regensburg, Regensburg, Germany. ${ }^{3}$ Institute of Paidopathology, Pediatric Tumor Registry, Christian-Albrecht's-University Kiel, Kiel, Germany. ${ }^{4}$ Institute of Human Genetics, Helmholtz Zentrum München, Neuherberg, Germany. ${ }^{5}$ Institute of Human Genetics, Technische Universität München, Munich, Germany.

\section{Received: 7 November 2017 Accepted: 21 February 2018}

\section{Published online: 02 March 2018}

\section{References}

1. Zimmermann A. The emerging family of hepatoblastoma tumours: from ontogenesis to oncogenesis. Eur J Cancer. 2005:41:1503-14.

2. Czauderna P, Lopez-Terrada D, Hiyama E, Haberle B, Malogolowkin MH, Meyers RL. Hepatoblastoma state of the art: pathology, genetics, risk stratification, and chemotherapy. Curr Opin Pediatr. 2014;26:19-28.

3. Meyers RL, Maibach R, Hiyama E, Haberle B, Krailo M, Rangaswami A, Aronson DC, Malogolowkin MH, Perilongo G, von Schweinitz D, et al. Riskstratified staging in paediatric hepatoblastoma: a unified analysis from the Children's Hepatic tumors International Collaboration. Lancet Oncol. 2017; 18:122-31

4. Zsiros J, Maibach R, Shafford E, Brugieres L, Brock P, Czauderna P, Roebuck D, Childs M, Zimmermann A, Laithier V, et al. Successful treatment of childhood high-risk hepatoblastoma with dose-intensive multiagent chemotherapy and surgery: final results of the SIOPEL-3HR study. J Clin Oncol. 2010;28:2584-90.

5. Cairo S, Armengol C, De Reynies A, Wei Y, Thomas E, Renard CA, Goga A, Balakrishnan A, Semeraro M, Gresh L, et al. Hepatic stem-like phenotype and interplay of Wnt/beta-catenin and Myc signaling in aggressive childhood liver cancer. Cancer Cell. 2008;14:471-84.

6. Sumazin $P$, Chen $Y$, Trevino LR, Sarabia SF, Hampton OA, Patel K, Mistretta TA, Zorman B, Thompson P, Heczey A, et al. Genomic analysis of hepatoblastoma identifies distinct molecular and prognostic subgroups. Hepatology. 2017;65:104-21.

7. Eichenmüller $M$, Trippel $F$, Kreuder M, Beck A, Schwarzmayr T, Häberle B, Cairo S, Leuschner I, von Schweinitz D, Strom TM, Kappler R. The genomic landscape of hepatoblastoma and their progenies with HCC-like features. J Hepatol. 2014;61:1312-20.

8. Beck A, Eberherr C, Hagemann M, Cairo S, Haberle B, Vokuhl C, von Schweinitz D, Kappler R. Connectivity map identifies HDAC inhibition as a treatment option of high-risk hepatoblastoma. Cancer Biol Ther. 2016;17:1168-76.

9. Regel I, Eichenmüller M, Joppien S, Liebl J, Häberle B, Müller-Höcker J, Vollmar A, von Schweinitz D, Kappler R. IGFBP3 impedes aggressive growth of pediatric liver cancer and is epigenetically silenced in vascular invasive and metastatic tumors. Mol Cancer. 2012;11:9.

10. Tomlinson GE, Kappler R. Genetics and epigenetics of hepatoblastoma. Pediatr Blood Cancer. 2012;59:785-92.

11. Felle M, Joppien S, Nemeth A, Diermeier S, Thalhammer V, Dobner T, Kremmer E, Kappler R, Langst G. The USP7/Dnmt1 complex stimulates the DNA methylation activity of Dnmt1 and regulates the stability of UHRF1. Nucleic Acids Res. 2011:39:8355-65.

12. Hermann A, Gowher H, Jeltsch A. Biochemistry and biology of mammalian DNA methyltransferases. Cell Mol Life Sci. 2004;61:2571-87.

13. Freitag M, Selker EU. Controlling DNA methylation: many roads to one modification. Curr Opin Genet Dev. 2005;15:191-9.

14. Bostick M, Kim JK, Esteve PO, Clark A, Pradhan S, Jacobsen SE. UHRF1 plays a role in maintaining DNA methylation in mammalian cells. Science. 2007; $317: 1760-4$
15. Karagianni P, Amazit L, Qin J, Wong J. ICBP90, a novel methyl K9 H3 binding protein linking protein ubiquitination with heterochromatin formation. Mol Cell Biol. 2008;28:705-17.

16. Bronner C, Fuhrmann G, Chedin FL, Macaluso M, Dhe-Paganon S. UHRF1 links the histone code and DNA methylation to ensure faithful epigenetic memory inheritance. Genet Epigenet. 2009;2010:29-36.

17. Unoki M, Nishidate T, Nakamura Y. ICBP90, an E2F-1 target, recruits HDAC1 and binds to methyl-CpG through its SRA domain. Oncogene. 2004;23: 7601-10.

18. Hashimoto $\mathrm{H}$, Horton JR, Zhang $\mathrm{X}$, Cheng X. UHRF1, a modular multidomain protein, regulates replication-coupled crosstalk between DNA methylation and histone modifications. Epigenetics. 2009;4:8-14.

19. Alhosin M, Omran Z, Zamzami MA, Al-Malki AL, Choudhry H, Mousli M, Bronner C. Signalling pathways in UHRF1-dependent regulation of tumor suppressor genes in cancer. J Exp Clin Cancer Res. 2016:35:174.

20. Ashraf W, Ibrahim A, Alhosin M, Zaayter L, Ouararhni K, Papin C, Ahmad T, Hamiche A, Mely Y, Bronner C, Mousli M. The epigenetic integrator UHRF1: on the road to become a universal biomarker for cancer. Oncotarget. 2017; 8:51946-62.

21. Eichenmüller M, Gruner I, Hagl B, Häberle B, Müller-Höcker J, von Schweinitz D, Kappler R. Blocking the hedgehog pathway inhibits hepatoblastoma growth. Hepatology. 2009;49:482-90.

22. Pfaffl MW. A new mathematical model for relative quantification in real-time RT-PCR. Nucleic Acids Res. 2001;29:e45.

23. Dobin A, Davis CA, Schlesinger F, Drenkow J, Zaleski C, Jha S, Batut $P$, Chaisson M, Gingeras TR. STAR: ultrafast universal RNA-seq aligner. Bioinformatics. 2013;29:15-21.

24. Anders S, Pyl PT, Huber W. HTSeq-a python framework to work with highthroughput sequencing data. Bioinformatics. 2015;31:166-9.

25. Love Ml, Huber W, Anders S. Moderated estimation of fold change and dispersion for RNA-seq data with DESeq2. Genome Biol. 2014;15:550.

26. Subramanian A, Tamayo P, Mootha VK, Mukherjee S, Ebert BL, Gillette MA, Paulovich A, Pomeroy SL, Golub TR, Lander ES, Mesirov JP. Gene set enrichment analysis: a knowledge-based approach for interpreting genomewide expression profiles. Proc Natl Acad Sci. 2005;102:15545-50.

27. Jung HJ, Byun HO, Jee BA, Min S, Jeoun UW, Lee YK, Seo Y, Woo HG, Yoon G. The ubiquitin-like with PHD and ring finger domains 1 (UHRF1)/DNA methyltransferase 1 (DNMT1) axis is a primary regulator of cell senescence. $J$ Biol Chem. 2017;292:3729-39.

28. Abu-Alainin W, Gana T, Liloglou T, Olayanju A, Barrera LN, Ferguson R, Campbell F, Andrews T, Goldring C, Kitteringham N, et al. UHRF1 regulation of the Keap1-Nrf2 pathway in pancreatic cancer contributes to oncogenesis. I Pathol. 2016:238:423-33.

29. Zhuo H, Tang J, Lin Z, Jiang R, Zhang X, Ji J, Wang P, Sun B. The aberrant expression of MEG3 regulated by UHRF1 predicts the prognosis of hepatocellular carcinoma. Mol Carcinog. 2016;55:209-19.

30. Nguyen LH, Robinton DA, Seligson MT, Wu L, Li L, Rakheja D, Comerford SA Ramezani S, Sun X, Parikh MS, et al. Lin28b is sufficient to drive liver cancer and necessary for its maintenance in murine models. Cancer Cell. 2014;26:248-61.

31. Vogelstein B, Papadopoulos N, Velculescu VE, Zhou S, Diaz LA Jr, Kinzler KW. Cancer genome landscapes. Science. 2013;339:1546-58.

32. Aguiar TFM, Carneiro TN, da Costa CML, Rosenberg C, da Cunha IW Krepischi ACV. The genetic and epigenetic landscapes of hepatoblastomas. Applied Cancer Research. 2017;37:20.

33. Ma J, Peng J, Mo R, Ma S, Wang J, Zang L, Li W, Fan J. Ubiquitin E3 ligase UHRF1 regulates p53 ubiquitination and p53-dependent cell apoptosis in clear cell renal cell carcinoma. Biochem Biophys Res Commun. 2015;464:147-53.

34. Yoo CB, Jones PA. Epigenetic therapy of cancer: past, present and future. Nat Rev Drug Discov. 2006;5:37-50

35. Ding G, Chen P, Zhang H, Huang X, Zang Y, Li J, Li J, Wong J. Regulation of ubiquitin-like with plant homeodomain and RING finger domain 1 (UHRF1) protein stability by heat shock protein 90 chaperone machinery. J Biol Chem. 2016:291:20125-35.

36. Seo JS, Choi YH, Moon JW, Kim HS, Park S-H. Hinokitiol induces DNA demethylation via DNMT1 and UHRF1 inhibition in colon cancer cells. BMC Cell Biol. 2017;18:14

37. Walker DA, Wyhs N, Giovinazzo H, Yegnasubramanian S, Nelson WG. Abstract 5390: development of a high-throughput screening assay to identify UHRF1 inhibitors via time-resolved fluorescence resonance energy transfer (TR-FRET). Cancer Res. 2014;74:5390. 
38. Unoki M, Brunet J, Mousli M. Drug discovery targeting epigenetic codes: the great potential of UHRF1, which links DNA methylation and histone modifications, as a drug target in cancers and toxoplasmosis. Biochem Pharmacol. 2009;78:1279-88.

39. Sidhu H, Capalash N. UHRF1: the key regulator of epigenetics and molecular target for cancer therapeutics. Tumour Biol. 2017;39:1010428317692205.

40. Zhou L, Shang Y, Jin Z, Zhang W, Lv C, Zhao X, Liu Y, Li N, Liang J. UHRF1 promotes proliferation of gastric cancer via mediating tumor suppressor gene hypermethylation. Cancer Biol Ther. 2015;16:1241-51.

41. Liu X, Ou H, Xiang L, Li X, Huang Y, Yang D. Elevated UHRF1 expression contributes to poor prognosis by promoting cell proliferation and metastasis in hepatocellular carcinoma. Oncotarget. 2017;8:10510-22.

Submit your next manuscript to BioMed Central and we will help you at every step:

- We accept pre-submission inquiries

- Our selector tool helps you to find the most relevant journal

- We provide round the clock customer support

- Convenient online submission

- Thorough peer review

- Inclusion in PubMed and all major indexing services

- Maximum visibility for your research

Submit your manuscript at www.biomedcentral.com/submit
Biomed Central 